\title{
La doble excepcionalidad antártica en tiempos de pandemia ${ }^{1}$
}

\author{
Cristian Lorenzo \\ Diego Navarro Drazich
}

Recibido: 30/11/2020

\author{
clorenzo@conicet.gov.ar \\ Centro Austral de Investigaciones Científicas (CADIC- \\ CONICET) \\ navarrodrazich@yahoo.com \\ Universidad Nacional de San Juan
}

Aceptado: 01/11/2021

Resumen: Si bien no hay contagios de COVID-19 en la Antártida, no quiere decir que lo que ocurre en el Continente Blanco esté absolutamente aislado de la realidad global, su dinámica y sus tendencias. Este virus impactó sobre actividades antárticas vinculadas al turismo, el sistema de toma de decisiones y las actividades científicas. Esos cambios complementan la excepcionalidad diplomática asociada a las negociaciones vinculadas con el Tratado Antártico y todo su sistema, que convirtieron al Continente Antártico y sus aguas circundantes, en una región dedicada a la paz y ciencia. Todo esto permite afirmar que predomina una doble excepcionalidad antártica en tiempos de pandemia.

Palabras clave: Sistema del Tratado Antártico; Políticas Antárticas; Geopolítica Antártica; Cooperación Antártica, COVID-19; Excepcionalidad Antártica; Turismo Antártico.

* Cómo citar: Lorenzo, C. y Navarro-Drazich, D. (2021). La doble excepcionalidad antártica en tiempos de pandemia. Relaciones Internacionales, 30(61), 139. https://doi.org/10.24215/23142766e139

${ }^{1}$ Los autores de este trabajo agradecen los comentarios recibidos por los evaluadores.

Editor: Juan Alberto Rial, Instituto de Relaciones Internacionales Facultad de Ciencias Jurídicas y Sociales (Universidad Nacional de La Plata)

\section{Entidad editora:Relaciones}

Internacionales, es una publicación del Instituto de Relaciones Internacionales (Facultad de Ciencias Jurídicas y Sociales (Universidad Nacional de La Plata Argentina) 


\title{
The double Antarctic exceptionality in times of pandemic
}

\section{Cristian Lorenzo ${ }^{2}$ Diego Navarro Drazich ${ }^{3}$}

\begin{abstract}
Although there are no COVID-19 infections in Antarctica, this does not mean that what happens on the White Continent is absolutely isolated from the global reality, its dynamics and trends. In fact, this virus has had an impact on Antarctic activities related to tourism, decision-making and scientific activities. These changes supplement the diplomatic exceptionality associated with negotiations related to the Antarctic Treaty and its entire system, which allow the Antarctic Continent and its surrounding waters to be a region devoted to peace and science. The point above allows us to claim that a double Antarctic exceptionality is predominant in times of pandemic.
\end{abstract}

Keywords: Antarctic Treaty System; Antarctic Policies; Antarctic Geopolitics; Antarctic Cooperation; COVID-19; Antarctic exceptionality; Antarctic Tourism

Este trabajo se realizó en el marco de dos proyectos: PID UNTDF "La política del Reino Unido hacia la Antártida, 2002-2019", financiado por la Universidad Nacional de Tierra del Fuego, Antártida e Islas del Atlántico Sur; y el PICT 2019 “¿Quién decide en tiempos de cambio climático? Política del Reino Unido hacia las áreas protegidas en la Antártida, 19982018", que se realiza con fondos de la Agencia Nacional de Promoción de la Investigación, el Desarrollo Tecnológico y la Innovación del Ministerio de Ciencia, Tecnología e Innovación.

\footnotetext{
${ }^{2}$ Investigador Adjunto del CONICET en el Centro Austral de Investigaciones Científicas (CADIC-CONICET). Docente-Investigador de la Universidad Nacional de Tierra del Fuego, Antártida, Islas del Atlántico Sur y Asuntos Internacionales, en el Instituto de Ciencias Polares, Ambiente y Recursos Naturales (ICPA). Doctor en Relaciones Internacionales, Universidad del Salvador. Magíster en Ciencia Política y Sociología, FLACSO Argentina. Licenciado en Relaciones Internacionales, Universidad del Salvador. Ushuaia, Argentina.

${ }^{3}$ Investigador adjunto del CONICET en la Universidad Nacional de San Juan. Profesor titular (a cargo) de Relaciones Internacionales en la Universidad Nacional de Cuyo, profesor titular (efectivo) en la Universidad del Aconcagua (Mendoza), profesor visitante de Globalización y América Latina de la Maestría en Relaciones Internacionales en la Universidad de la Empresa (Montevideo) y profesor en el Doctorado en Geografía de la Universidad Nacional de San Juan. Doctor en Relaciones Internacionales, Universidad del Salvador.
} 


\section{Introducción}

El 11 de marzo de 2020, el Director General de la Organización Mundial de la Salud (OMS) declaró al brote de COVID-19 como una pandemia (Reuters, 2020a). En aquel momento, en el mundo había 118.000 personas con coronavirus y 4.291 fallecidas a causa de esta enfermedad (OMS, 2020). Durante varios meses, la Antártida permaneció libre de este virus, hasta el 17 de diciembre, cuando la Armada chilena informó que la tripulación del buque Sargento Aldea estaba en cuarentena4. El mes anterior, esa embarcación había estado en la base antártica chilena Bernardo O’Higgins y el 21 de diciembre el Ejército de Chile comunicó que 36 personas se habían contagiado en dicha base (Senado de la República de Chile, 2021). Dada esta situación, nos preguntamos: ¿cómo impacta la pandemia del COVID-19 al Sistema del Tratado Antártico?

El COVID-19 ha impactado en las regiones polares (Forrester, 2021). En particular, despierta suspicacias sobre el comportamiento de algunos países en la Antártida (Feiger, Wilson, 2020). En la literatura especializada, se ha examinado su relación con las decisiones, la logística, la ciencia, el turismo y la pesca (Dodds \& Hemmings, 2020; Hemmings, 2020; Hughes \& Convey, 2020), y hasta fue considerado como uno de los factores relevantes para los escenarios futuros de la región antártica (Frame et al., 2021). Estos trabajos aportan elementos para reflexionar sobre el presente y el futuro del Continente Blanco, desde perspectivas del Norte Global.

Las disciplinas están atravesadas por relaciones de poder a nivel mundial (Beigel, 2018). En el caso de las Relaciones Internacionales predomina una versión estadounidense (Tickner et al., 2012). Esto no quiere decir que sea el único marco posible de referencia. Este trabajo se ubica en la tradición del pensamiento latinoamericano en Relaciones Internacionales (Bernal-Meza, 2016; Devés \& Álvarez, 2020). En este contexto, partimos del siguiente presupuesto: "todo conocimiento es el resultado de unas premisas y unas preguntas situadas espacio-temporalmente" (Seitz, 2011). Así, damos por supuesto que hay tradiciones en nuestra disciplina y que la producción de conocimiento necesita situar el lugar de enunciación geográfico y hermenéutico.

Pare este trabajo, se relevó información sobre Programas Nacionales Antárticos, medios internacionales de prensa, foros antárticos, y literatura especializada, teniendo en cuenta la pregunta central de investigación. El análisis no fue realizado desde un marco teórico prefijado (Cervo, 2008), sino desde la inducción analítica (Denzin, 2007). Es por ello que las afirmaciones empíricas se fueron ajustando mediante sucesivas observaciones.

La constatación central de este trabajo es que desde la declaración de la pandemia del COVID-19 por la OMS predomina una doble excepcionalidad antártica, que impacta sobre las actividades vinculadas al turismo, el sistema de toma de decisiones y la ciencia.

\footnotetext{
${ }^{4}$ Antártida se refiere a la región que está al sur de los $60^{\circ}$ de latitud sur, incluidas todas las barreras de hielo, tal como es definido por el Tratado Antártico.
} 
Este trabajo está organizado de la siguiente manera. En primer lugar, se examinan tres ámbitos relacionados con esta región: las actividades turísticas, el sistema de toma de decisiones y las actividades científicas. Todo esto se toma, luego, de base para explorar conceptualmente si se puede afirmar que estamos ante un nuevo rostro de la excepcionalidad antártica.

\section{El turismo antártico}

La pandemia del COVID-19 impacta al sector público y privado del turismo de cruceros antárticos. Antes del inicio de la temporada 2020-2021, las autoridades portuarias en la provincia de Tierra del Fuego, Antártida e Islas del Atlántico Sur establecieron un protocolo para evitar la propagación del virus y, en este marco, una serie de medidas para las operaciones de cruceros desde el Puerto Provincial en Ushuaia ${ }^{5}$. Dicho protocolo fue elaborado en conjunto con el Comité Operativo de Emergencia (COE), la Dirección Provincial de Puertos y el Instituto Fueguino de Turismo (INFUETUR) (Diario del Fin del Mundo, 2020). Por otro lado, la pandemia también impactó sobre decisiones comerciales. En la temporada referida, varias empresas de cruceros cancelaron sus operaciones (IAATO, 2020).

A pesar de los protocolos, el riesgo siempre existe. El Greg Mortimer, un crucero operado por Aurora Expeditions, tuvo más de cien personas contagiadas a bordo durante marzo de 2020 (El Telégrafo, 2020). Esta nave desembarcó en el Puerto de Montevideo a través de un Corredor Humanitario, organizado por la Cancillería de Uruguay, en coordinación con otros ministerios. Primero, lo hicieron aquellos que necesitaban atención personalizada por sus condiciones de salud; el resto tuvo que esperar a que el test de coronavirus les diera negativo. Para descender, tenían que tener un avión disponible para embarcar hacia sus países de residencia. No podían permanecer en territorio uruguayo (Ministerio de Relaciones Exteriores de la República Uruguay, 2020).

El caso del Greg Mortimer también manifiestó un nuevo ángulo de la situación. Si bien la relación entre las empresas que operan en la Antártida y sus tripulantes está regulada por un contrato, no está exenta de conflictos. Como ejemplo podemos considerar la denuncia del médico responsable a bordo, quien objetó la decisión de la empresa de operar cuando las recomendaciones internacionales eran no hacerlo y denunció las presiones que recibió al confeccionar los formularios que serían evaluados por las autoridades sanitarias del lugar de desembarco. El profesional también manifestó públicamente su disconformidad acerca de la decisión de la empresa de dirigirse a Islas Canarias (España) y no permitir a sus tripulantes regresar a sus hogares desde Uruguay, tal como estaba convenido (El Observador, 2020)

\footnotetext{
${ }^{5}$ Cabe subrayar que a nivel mundial, Ushuaia es reconocida como la puerta de entrada más cercana a la Antártida.
} 
En términos generales, el turismo de cruceros antárticos fue impactado por el COVID-19 de múltiples maneras, dados los actores implicados en situaciones de emergencia, las instancias de planificación en materia de prevención y evacuación, y las relaciones laborales entre sus tripulantes y las empresas de cruceros. Esto demuestra la vulnerabilidad del negocio de los operadores turísticos antárticos ante esta situación generada por los riesgos de contagio.

\section{El sistema de toma de decisiones}

La pandemia del COVID-19 afectó el normal desarrollo de reuniones multilaterales sobre temas antárticos. El 12 de marzo de 2020 se decidió cancelar la Reunión Consultiva y del Comité de Protección Ambiental previstas en Helsinki entre el 25 de mayo y el 4 de junio de ese año. Así lo comunicó la Cancillería de Finlandia (Ministry of Foreign Affairs of Finland, 2020). Al día siguiente, esta decisión fue informada por la Secretaría del Tratado Antártico (Secretaría del Tratado Antártico, 2020a). En realidad, hubo más reuniones presenciales que no se llevaron adelante, como la del Comité Organizador del SCAR (Comité Científico de Investigación Antártica) y del COMNAP (Consejo de Administradores de Programas Antárticos Nacionales), previstas para junio en Hobart (Australia) (Secretaría del Tratado Antártico, 2020b).

Algunas reuniones pasaron a desarrollarse en formato virtual, como fue el caso de las anuales de la Comisión, perteneciente a la Convención para la Conservación de los Recursos Vivos Marinos, entre el 27 y el 30 de octubre de 2020 (CCAMLR, 2020a). Esto fue posible, porque lo permite su reglamento en el artículo 7 (CCAMLR, 2020b). Con relación a sus implicancias, hay un aspecto de las relaciones personales que resulta fundamental en estos ámbitos y es, precisamente, por dónde pasan las negociaciones antárticas. Esto es lo que también observaron Doods y Hemmings al respecto:

Por procedimiento, el Sistema del Tratado Antártico funciona como un régimen basado en la presencialidad, donde las reuniones cara a cara y los pasillos son priorizados por los representantes diplomáticos en las reuniones anuales - las decisiones son realizadas en estos eventos y, en consecuencia, no es tal vez sorprendente. Las decisiones tomadas por consenso también demandan altos nivel de emocionalidad, así como también, horas de trabajo. Las relaciones son hechas y reelaboradas en las reuniones (Doods \& Hemmings, 2020: 532).

De hecho hay una cuestión más que observan:

La gobernanza antártica no ha adoptado las tecnologías de trabajo digital en relación con cuestiones de fondo de una manera que otros regímenes internacionales y foros intergubernamentales, como el Consejo Ártico (Doods \& Hemmings, 2020: 533).

A modo de ejemplo, una cuestión relevante para la agenda antártica es la creación de un área marina protegida alrededor de la Península Antártica, impulsada por Argentina y Chile (CCAMLR, 2020b). Sin embargo, la posibilidad de su establecimiento todavía requiere 
conjugar intereses científicos, pesqueros, conservación y geopolíticos (Lorenzo, 2020).

Hasta el momento, se crearon dos áreas marinas protegidas: la primera fue en las Islas Orcadas del Sur ${ }^{6}$ (CCAMLR, 2009) y la segunda, en el Mar de Ross ${ }^{7}$ (CCAMLR, 2016). Cada una de estas decisiones estuvo respaldada científicamente, utilizando la mejor ciencia disponible. Es por este motivo que esta última juega un rol clave en los procesos de toma de decisiones relacionados con la región antártica.

Las políticas de prevención asociadas al COVID-19 y sus efectos sobre el sistema de toma de decisiones abren interrogantes sobre si se modificarán las formas pautadas para la realización de reuniones y la toma de decisiones sobre cuestiones sustantivas. En 2021, las Reuniones Consultivas fueron organizadas por Francia en forma virtual, pero ¿qué pasará para las próximas? ¿En qué medida puede afectar cuando hay que tomar decisiones que requieren complejas negociaciones?

\section{Actividades científicas}

¿Cómo impactó el COVID-19 sobre la ciencia antártica? Las exploraciones realizadas nos permiten constatar que hay una dimensión multilateral que se retroalimenta con la planificación de los programas nacionales antárticos. El 12 de marzo de 2020 el Comité Ad Hoc de COMNAP comenzó a trabajar en el desarrollo de recomendaciones para los miembros de los Programas Antárticos Nacionales. Uno de las indicaciones era cómo proceder ante casos de contagios de coronavirus (Christchurch Antarctic Office, 2020). Dichas indicaciones fueron complementadas durante los meses siguientes desde esta organización multilateral. Los nuevos dilemas pasaban por saber cuáles iban a ser los recursos médicos disponibles, y definir los procedimientos y mecanismos de comunicación entre las autoridades sanitarias (COMNAP, 2020). El COVID-19 también impactó sobre el sistema de cooperación regional antártico. De hecho, en la Reunión de Administradores de Programas Latinoamericanos (RAPAL), el protocolo para la logística fue uno de los temas de la agenda (Telam, 2020).

En otro nivel, los programas antárticos nacionales buscan evitar contagios en el Continente Blanco: "Para todas las naciones trabajando en Antártida, el principal objetivo es mantener el virus fuera del hielo", dijo Christine Wesche, coordinadora logística del Programa Antártico Nacional de Alemania (National Geographic, 2020). De hecho, al operar en la región antártica, las actividades científicas están supeditadas a las medidas de prevención. Como ejemplo, en junio de 2020 la Fuerza Aérea Argentina realizó un cruce aéreo desde Río Gallegos hacia Base Marambio para abastecer a las bases argentinas y apoyar logísticamente a las actividades científicas. Dicho cruce se rigió por normativa establecida

\footnotetext{
${ }^{6}$ Medida de conservación 91-05 del 2009.

${ }^{7}$ Medida de conservación 93-05 del 2016.
} 
por el Comando Conjunto Antártico, que lo restringe a una serie de situaciones:

situaciones de carácter crítico y en los que la ejecución del traslado sea determinante para la solución de un grave problema de sanidad, científico o logístico, donde su no resolución desencadene consecuencias no aceptables para el Comando Conjunto Antártico (Ministerio de Defensa de la República Argentina, 2020a).

El riesgo de contagios en la Antártida motivó la consulta entre programas antárticos nacionales, para evaluar escenarios futuros y redefinir las prioridades en el desarrollo de investigaciones científicas. Todavía es difícil evaluar el impacto de estas decisiones, como dijo Stephanie Short, jefa de la logística antártica de National Science Foundation (Heidt, 2020). Los Programas Antárticos planifican escenarios posibles, contemplando cambios inesperados durante el desarrollo de sus actividades antárticas (British Antarctic Survey, 2020). Las prioridades se redefinen, la cantidad de científicos que van a la Antártida se reduce notablemente y se establecen estrictos cumplimientos de protocolos de aislamiento, con anterioridad al viaje a la región antártica. Para ilustrar, se puede hacer referencia al procedimiento del Ministerio de Defensa de la República Argentina:

(...) el objetivo principal es cumplir un estricto protocolo sanitario que consiste en el aislamiento preventivo durante 14 días a cada dotación que está por viajar. A cada uno de sus integrantes, el primer día se le hace un test de PCR (hisopado para detectar el coronavirus) y durante esas dos semanas se les efectúan controles médicos, los que concluyen el día 14 con otro test de PCR, y cuyo resultado no detectable le posibilita, finalmente, el ingreso seguro a la Antártida (Ministerio de Defensa de la República Argentina, 2020b).

Durante la pandemia, hay algunas investigaciones antárticas que se mantienen. En general, son las de largo plazo, cuya interrupción podría impactar decisivamente sobre la muestra de los datos y las conclusiones. También, hay otras que fueron pospuestas. En algunos casos, se pueden absorber por el tiempo de desarrollo del proyecto, pero en otros, cuando la duración es más acotada, el costo es alto (Science Media Centre, 2020). Desde los Programas Antárticos el mensaje que dan es que la ciencia antártica no se interrumpió en su totalidad. Como lo comunicó el Programa Antártico de Australia, sus científicos mantenían sus actividades en forma regular, como lo demuestran las participaciones en congresos y las publicaciones en revistas científicas con referato a nivel internacional (Australian Antarctic Division, 2020).

La dimensión personal también está implicada cuando se trata de las relaciones entre ciencia y logística cuando hay brotes de COVID-19. La realidad epidemiológica que les toca vivir a quienes están en la Antártida es muy diferente al resto del mundo. La conectividad les permite estar actualizados sobre las situaciones de sus familias, de sus países y de lo que ocurre en el mundo (Daily Mirror, 2020). Sin embargo, esta situación despierta distintas lecturas personales, como interpretar que se está en un aislamiento dentro de otro aislamiento (Reuters, 2020b) y estar preocupados por la salud de sus familias que están en sus países de origen (Newburger, 2020). 


\section{5. ¿Hacia una nueva excepcionalidad antártica?}

Examinamos ahora si, en términos conceptuales, estamos ante una nueva excepcionalidad antártica. Como primera aproximación a nivel diplomático, la región antártica es reconocida como un ejemplo mundial de paz. En 1959, doce Estados con diferentes intereses territoriales sobre el Continente Blanco alcanzaron un acuerdo que todavía perdura y fundamenta el actual status quo de esta parte del mundo. Esta visión está asociada a una mirada del derecho internacional. De acuerdo a esta línea, Abruza (2013) señala que:

Los cincuenta años de vigencia del Tratado prueban que ha sido el resultado de uno de los más exitosos ejercicios de diplomacia multilateral del siglo xx, que se apoyó desde el inicio en la ciencia y, más tarde, en la protección del medio ambiente antártico y facilitó un valioso e intenso proceso de cooperación internacional para mantener y consolidar la paz en la región antártica (p. 142).

De todos modos, hay desafíos asociados a los instrumentos legales vinculados a la región antártica. Con relación a esta perspectiva, Ferrada puntualiza algunas ideas sobre el Protocolo de Madrid:

(...) es necesario establecer una distinción entre perspectivas diplomáticas, legales y prácticas. Desde el punto de vista diplomático, es el mejor logro. Su negociación fue muy exitosa, aun considerando los desacuerdos y problemas geopolíticas que ha tenido que enfrentar. Desde un punto de vista legal, el Protocolo ha asegurado importantes logros y ha sido el punto de partida para otras áreas del derecho internacional, como la regulación de actividades minerales en el fondo del mar. Pero, al mismo tiempo, tiene una significativa debilidad, como la falta de mecanismos de control efectivo o los de un régimen de responsabilidad (Ferrada, 2012)

Por otro lado, se pueden destacar otros aspectos de la excepcionalidad antártica. En línea con una perspectiva histórico-global, Portella Sampaio (2019) señala que la "excepción antártica" necesita comprenderse en la historia de la sociedad internacional. Y esto, sin duda, requiere reconocer que la configuración actual alrededor del Tratado Antártico y su sistema se insertan en una historia de larga duración. Su singularidad está en que, al sur de los $60^{\circ}$ de latitud Sur, no se replican de la misma forma las lógicas globales de conflicto asociadas a la sociedad internacional

[y] el Tratado Antártico encarnó este momento de la creación política: se creó un marco de gobernanza mediante una solución sin precedentes para evitar la replicación de la localización de las normas de soberanía y territorialidad de la sociedad internacional. En la Antártida, el Tratado proporcionó un mecanismo de toma de decisiones donde la autoridad puede desplegarse sin requerir una definición clara de quién detiene su monopolio. Y el consenso fue el procedimiento elegido para permitir esta forma de despliegue de autoridad (p. 110).

Este conjunto de elementos conforman la excepcionalidad antártica diplomática. Es- 
ta realidad todavía persiste en un contexto en el que la Antártida tiene un valor estratégico para países sudamericanos como Argentina y Chile (Estenssoro, 2020) y en el que se producen cambios ambientales asociados al cambio climático en la Antártida.

La pregunta ahora es si -con este trasfondo- los impactos del COVID-19 sobre las actividades humanas en la Antártida constituyen una nueva forma de esta excepcionalidad. El diccionario de la Real Academia Española presenta dos acepciones para definir el significado del término "excepcional". La primera dice "que constituye excepción de la regla común" y la segunda, "que se aparta de lo ordinario, o que ocurre rara vez" (DRAE, 2020). Si tomamos en cuenta estas dos posibilidades, el impacto del coronavirus sobre el sistema de toma de decisiones, las actividades científicas y las operaciones de cruceros cubren ambas acepciones: ocurrió por primera vez desde la firma del Tratado Antártico, impactó sobre distintos ámbitos relacionados con la Antártida y alteró su normal desarrollo. Entonces, la reconfiguración originada por los impactos del COVID-19 sobre las cuestiones antárticas se asocia con una doble excepcionalidad. Dados los riesgos que existen asociados a los casos de contagios en la región antártica, resulta fundamental sostener esta doble excepcionalidad en el Continente Blanco.

\section{Conclusiones: nuevo rostro de la excepcionalidad antártica}

En este trabajo partimos con una pregunta: ¿Cómo impacta la pandemia del COVID19 al Sistema del Tratado Antártico? A partir de las distintas exploraciones realizadas, constatamos que predomina una doble excepcionalidad antártica en tiempos de pandemia y mostramos cómo se manifiesta. La diplomática es la más reconocida, porque convirtió a esta región del planeta en sinónimo de paz y cooperación desde 1959. Pero no es la única. Dicha excepcionalidad es complementada con una situación sanitaria dominante que muestra que lo que pasa al sur de los 60 grados de latitud sur no está absolutamente desconectado de realidades internacionales asociadas al despliegue del virus y las políticas de prevención.

La pandemia impacta sobre el turismo de cruceros antárticos, a través de la planificación y la coordinación, las distintas instancias de planificación asociadas a la prevención y la evacuación; y obre las relaciones laborales entre las empresas que operan en cruceros y su personal de a bordo.

También lo hace sobre el sistema de toma de decisiones que organiza y hace posible la protección y conservación de la Antártida a nivel multilateral. La emergencia de este virus y su rápida propagación en el mundo motivaron la cancelación de reuniones y algunas de ellas pasaron a formato virtual.

En tercer y último lugar, la pandemia impacta sobre el desarrollo de la ciencia antártica. A nivel personal, cuando se trata de quienes realizan proyectos de investigación en el Continente Blanco; a nivel institucional, en materia de planificación y definición de prioridades de los Programas Antárticos Nacionales; y a nivel multilateral, en la coordinación entre los Programas Nacionales Antárticos.

Este conjunto de interacciones complementan la excepcionalidad diplomática aso- 
ciada al Tratado Antártico y todo su sistema, los que permiten que el Continente Antártico y sus aguas circundantes formen parte de una región dedicada a la paz y la ciencia. Es por ello que observamos que hay doble excepcionalidad antártica en tiempos de pandemia.

Surgen ahora una serie de preguntas: ¿Se podrá mantener esta predominante excepcionalidad por mucho tiempo más? ¿En qué medida se relaciona con la resiliencia del Sistema el Tratado Antártico? ¿Cuáles son las oportunidades y los desafíos que presenta el impacto del COVID-19 en la Antártida a la cooperación antártica latinoamericana? Estos interrogantes podrían dar lugar a nuevas investigaciones.

\section{Referencias}

Abruza, A. (2013). “El Tratado Antártico y su sistema”, Boletín del Centro Naval, 836.

Australian Antarctic Division (2020). "Science under COVID-19", Jul 22. https://www.antarctica.gov.au/news/2020/science-under-covid-19/

Beigel, F. (2018). "Las relaciones de poder en la ciencia mundial”, Revista Nueva Sociedad, 274. https://nuso.org/articulo/las-relaciones-de-poder-en-la-ciencia-mundial/

Bernal-Meza, R. (2016). “Contemporary Latin American thinking on International Relations: theoretical, conceptual and methodological contributions", Revista Brasileira de Política Internacional, 59, 1.

British Antarctic Survey (2020). “Update on 2020/21 Antarctic field season: responding to COVID-19 pandemic", Aug 7. https://www.bas.ac.uk/media-post/update-on2020-21-antarctic-field-season-responding-to-covid-19-pandemic/

CCAMLR, Commission for the Conservation of Antarctic Marine Living Resources (2009). Medida de conservación 91-03 (2009). https://www.ccamlr.org/en/measure91-03-2009

CCAMLR, Commission for the Conservation of Antarctic Marine Living Resources (2016). Medida de conservación 91-05 (2016). https://www.ccamlr.org/en/measure91-05-2016

CCAMLR, Commission for the Conservation of Antarctic Marine Living Resources (2020a). Meeting of Antarctic experts comes to a close. https://www.ccamlr.org/en/news/2020/meeting-antarctic-experts-comesclose

CCAMLR, Commission for the Conservation of Antarctic Marine Living Resources (2020b). "Informe de la trigésima novena reunión de la Comisión. Reunión virtual, 26 al 30 de octubre de 2020", Noviembre 6. https://www.ccamlr.org/es/system/files/s-cc-39-prelim-v1.2.pdf

Cervo, A. (2008). "Conceitos em Relações Internacionais", Revista Brasileira de Política Internacional, 55, 2, pp. 8-25.

Christchurch Antarctic Office (2020). "Update from Michelle Rogan-Finnemore, COMNAP Executive Secretary", Mayo 25. 
https://www.christchurchnz.com/news/update-from-michelle-roganfinnemore-comnap-executive-secretary

COMNAP, Council of Managers of National Antarctic Programs (2020). "Responses received from DNA from the Terra del Fuego province, Argentina", Junio 26. https://www.comnap.aq/wp-content/uploads/2020/06/COVID-ProcedureTierra-del-Fuego-26-June-2020.pdf

Daily Mirror (2020). "Scientist describes 'surreal' life on only coronavirus-free continent in the world", Abril 17. https://www.mirror.co.uk/science/scientist-antarcticadescribes-surreal-life-21884294

Denzin, N. (2007). “Analytical Induction”. In: Ritzer, G. The Blackwell Encyclopedia of Sociology.https://doi.org/10.1002/9781405165518.wbeosa048

Devés, E.; Álvarez, S. T. (Eds.). (2020). Problemáticas internacionales y mundiales desde el pensamiento latinoamericano. Teoría, Escuelas, Conceptos, Doctrinas, Figuras. Ariadna Ediciones.

Diario del Fin del Mundo. 2020. "Protocolo con vuelos encapsulados para turistas que vayan a la Antártida", 14 de octubre. https://www.eldiariodelfindelmundo.com/noticias/2020/10/14/88808protocolo-con-vuelos-encapsulados-para-turistas-que-vayan-a-la-antartida

Dodds, K., \& Hemmings, A. D. (2020). "Antarctic diplomacy in a time of pandemic". The Hague Journal of Diplomacy, 15(4), 530-541.

DRAE, Diccionario de la Real Academia Española, "excepcional". https://dle.rae.es/excepcional?m=form

Doods, K.; Hemmings, A. (2020). "Antarctic Diplomacy in a Time of Pandemic". The Hague Journal of Diplomacy, 15, 4: 530-541.

El Observador. 2020. “Denuncian que presionaron al médico del Greg Mortimer para que minimizara gravedad del brote", 4 de mayo.

https://www.elobservador.com.uy/nota/denuncian-presiones-sobre-medicodel-greg-mortimer-para-minimizar-gravedad-del-brote-202054134038

El Telégrafo. 2020. "Médico del crucero Greg Mortimer plantea una dramática situación", 8 de mayo. https://www.eltelegrafo.com/2020/05/medico-del-crucero-gregmortimer-plantea-una-dramatica-situacion/

Estenssoro, F. (2020). "Challenges of Latin America in the Global Environmental Geopolitics of the Twenty-First Century", en, Lorenzo, C. (Edit), Latin America in Times of Global Environmental Change. Springer, pp. 15-29.

Frame, B., Yermakova, Y., Flamm, P., Nicklin, G., De Paula, G., Badhe, R., \& Tuñez, F. (2021). "Antarctica's Gateways and Gatekeepers: Polar scenarios in a polarising Anthropocene". The Anthropocene Review, 20530196211026341.

Ferrada, V. (2012). "Antarctic Environment Protocol Challenges and Achievements: 20 Years in Force". https://www.ejiltalk.org/antarctic-environment-protocolchallenges-and-achievements-20-years-in-force/ 
Feiger, L., Mara Wilson (2020). "The Countries Taking Advantage of Antarctica During the Pandemic", The Atlantic, May 15.

https://www.theatlantic.com/politics/archive/2020/05/antarctica-greatpower-competition-australia-united-states-britain-russia-china-arctic/611674/ Forrester, N. (2021). "What polar researchers have learnt from the pandemic", Nature, July 6, doi.org/10.1038/d41586-021-01861-8

Heidt, A. (2020). “Coronavirus Precautions Stall Antarctic Field Research”, June 15. https://www.the-scientist.com/news-opinion/coronavirus-precautions-stallantarctic-field-research-67636

Hemmings, A. D. (2020). "Antarctic Governance in a Time of Coronavirus". ANZSIL Perspective, 13, 3-5.

Hughes, K.; Convey, P. (2020). "Implications of the COVID-19 pandemic for Antarctica", Antarctica Science, 32, 6, 426-439, doi:10.1017/S095410202000053X.

IAATO (2020). Asociación Internacional de Operadores Turísticos de la Antártida, “IAATO’s Response To COVID-19", September. https://iaato.org/wpcontent/uploads/2020/09/IAATO-Response-to-COVID-19-September-2020.pdf

Lorenzo, C. (2020). "La política detrás de la ciencia: la protección y conservación de recursos vivos marinos en la Antártida, 2005-2008", Revista Relaciones Internacionales, Estrategia y Seguridad, 15,1, 117 - 127. https://doi.org/10.18359/ries.4268

Ministerio de Defensa de la República Argentina (2020a). "Bajo un estricto protocolo sanitario, un avión Hércules realizó el primer cruce a la Antártida desde que se declaró la pandemia por COVID-19", 5 de junio de 2020. https://www.argentina.gob.ar/noticias/bajo-un-estricto-protocolo-sanitarioun-avion-hercules-realizo-el-primer-cruce-la-antartida

Ministerio de Defensa de la República Argentina (2020b). "La edición 117ạ de la Campaña Antártica de Verano 2020-2021 se realizará bajo un estricto protocolo sanitario", 14 de octubre. https://www.argentina.gob.ar/noticias/la-edicion-117ade-la-campana-antartica-de-verano-2020-2021-se-realizara-bajo-un-estricto

Ministerio de Relaciones Exteriores de la República de Uruguay (2020). “El Canciller anunció la última operación del Corredor Humanitario: el crucero Greg Mortimer", 3 de abril. https://www.gub.uy/ministerio-relacionesexteriores/comunicacion/noticias/canciller-anuncio-ultima-operacion-delcorredor-humanitario-crucero-greg

Ministry of Foreign Affairs of Finland (2020). "XLIII Antarctic Treaty Consultative Meeting 25.5 -4.6.2020, Helsinky, Finland", 12 March. https://um.fi/atcm43.

National Geographic (2020). "Antarctica is the last continent without COVID-19. Scientists want to keep it that way", Agosto 10.

https://www.nationalgeographic.co.uk/environment-andconservation/2020/08/antarctica-is-the-last-continent-without-covid-19scientists 
Newburger, E. (2020) "Antarctica, the only continent without coronavirus, braces for summer rotation", Sep 27. https://www.cnbc.com/2020/09/27/antarctica-the-onlycontinent-without-coronavirus-braces-for-summer.html

OMS, Organización Mundial de la Salud (2020). “Alocución de apertura del Director General de la OMS en la rueda de prensa sobre la COVID-19 celebrada el 11 de marzo de 2020", Marzo 11. https://www.who.int/es/dg/speeches/detail/whodirector-general-s-opening-remarks-at-the-media-briefing-on-covid-19---11march-2020

Portella Sampaio, D. (2019). "The Antarctic exception: how science and environmental protection provided alternative authority deployment and territoriality in Antarctica", Australian Journal of Maritime \& Ocean Affairs, 11, 2, 107-119. https://www.tandfonline.com/doi/full/10.1080/18366503.2019.1589899

Reuters (2020a). "Hurtigruten cancels Jan-March Antarctica cruises because of pandemic", Oct 22. https://www.reuters.com/article/hurtigruten-cancellationidUSL8N2HD49P

Reuters (2020b). "Isolated within isolation': Keeping the coronavirus out of Antarctica", Apr 15. https://www.japantimes.co.jp/news/2020/04/15/world/science-healthworld/coronavirus-antarctic/

Science Media Centre (2020). "Antarctic science shrunk for COVID-19 - Expert Reaction", Junio 9. https://www.sciencemediacentre.co.nz/2020/06/09/38798/

Secretaría del Tratado Antártico (2020a). “Cancelación de la RCTA XLIII y CPA XXIII”, 13 de marzo. https://www.ats.aq/devph/es/news/176

Secretaría del Tratado Antártico (2020b). "Se cancelaron las reuniones del SCAR y el COMNAP 2020 “, 19 de marzo, https://www.ats.aq/devph/es/news/177

Senado de la República de Chile (2021). "COVID-19 en la Antártida: senadores escuchan explicaciones del Ejecutivo pero critican medidas preventivas", 6 de enero, https://www.senado.cl/covid-19-en-la-antartida-senadores-escuchanexplicaciones-del-ejecutivo/senado/2021-01-06/091439.html

Telam (2020). “Argentina y Chile fijan protocolos conjuntos en operaciones y bases antárticas ante la pandemia", Mayo 27.

https://www.telam.com.ar/notas/202005/469050-argentina-y-chile-fijanprotocolos-conjuntos-en-operaciones-y-bases-antarticas-ante-lapandemia.html

Seitz, M. (2011). “Relaciones Internacionales desde América Latina”, en M. Langón; \& C. Lértora Mendoza (Eds.), La primera década del siglo XXI. Balance y perspectivas. XV Jornadas de Pensamiento Filosófico. FEPAI, 1-15.

Tickner, A., Cepeda, C., Bernal, J. (2012). “Enseñanza, Investigación y Política Internacional (TRIP) en América Latina”. Documentos del Departamento de Ciencia Política, Universidad de Los Andes, Bogotá. 